\title{
Entropy based Robust Watermarking Scheme using Hadamard Transformation Technique
}

\author{
Franklin Rajkumar.V \\ MS Software Engineering \\ School of Information Technology \\ and Engineering \\ VIT University \\ Vellore-India
}

\author{
Manekandan.GRS \\ MS Software Engineering \\ School of Information Technology \\ and Engineering \\ VIT University \\ Vellore-India
}

\author{
V.Santhi \\ Associate Professor \\ School of Computing Science \\ and Engineering \\ VIT University \\ Vellore-India
}

\begin{abstract}
As a novel watermarking algorithm is required to protect copy rights of digital data entropy based robust watermarking scheme using Hadamard transformation technique is proposed in this paper. The proposed technique can hide an entire image or pattern as a watermark directly into the original image. As the quality of the image is to be preserved the entire image is not altered for embedding, instead few blocks are used based on the size of the watermark and information content of an image block. To reduce the computational complexity of the proposed algorithm Hadamard transformation is used for converting cover image from spatial domain to transform domain. The proposed algorithm is tested with Lena grey scale image of size $256 \times 256$ and watermark of size $64 \times 64$ using mat lab software. The experimental results show that the proposed scheme is robust to random noise addition attack, resize attack and cropping attack.
\end{abstract}

\section{General Terms}

Image Processing, Multimedia Security, Digital Watermarking

\section{Keywords}

Digital Watermarking, Hadamard transform based watermarking, Entropy based watermarking, transform domain watermarking.

\section{INTRODUCTION}

With the advent of the Internet in this digital era, there is a forcing need for copyright enhancement schemes that protect copyright ownership of digital media. Watermarking is one of the multimedia authentication techniques [1]. Watermarking is the process of embedding a piece of digital information into any multimedia data such as an image, audio or video file for the purpose of authentication. Watermarks can be embedded in the pixel/spatial domain or a transform domain [2]. In spatial domain, the watermark is embedded directly by modifying the intensity values of pixels. In frequency domain, the watermark is embedded by changing the frequency coefficients. To transform image into frequency domain, the transformation techniques such as discrete wavelet transformation (DWT), discrete cosine transformation (DCT), discrete Hadamard transformation and discrete Fourier transformation are used. Spatial domain watermarking technique is easier and its computing speed is high, than transform domain watermarking. But the disadvantage is that it is not robust against common image processing operations. Transform domain techniques are introduced to increase the robustness of the digital media.
An effective watermark should have the following characteristics:

a) It should be perceptually invisible or its presence should not interfere with the work being protected [3, 4].

b) It should be robust to common signal processing operations such as sharpening, dithering, resizing, compression, noise addition and so on $[5,6]$.

The most important uses of watermark include copyright protection, authentication and disabling unauthorized access to the contents [7].

In section 1 introduction about the necessity of watermarking is given. Section 2 discuss briefly about the review of related works. In section 3 and 4 discusses about preliminaries of transformation techniques and entropy. In Section 5 proposed algorithm is given in detail as step by step procedure discusses in detail. Section 6 tells about the performance evaluation of proposed technique. Proposed technique is concluded in section 7. Finally, in section 8 all the papers which are referred are mentioned.

\section{REVIEW OF RELATED WORKS}

B. J. Fnlkowski et al embedded a watermark using multiresolution Hadamard and complex Hadamard transforms in a grey scale image [8].They segmented the multiresolution Hadamard transformed image into numerous $8 * 8$ blocks and then applied forward complex Hadamard transformation. This technique is robust to JPEG compression up to $10 \%$ quality factor, successive watermarking effects, and common signal processing operations such as dithering distortion (25\%), image resizing, cropping and scaling $(56.25 \%)$. Bogdan J. et al embedded a watermark using multiresolution and two dimensional complex Hadamard transformation techniques [9]. The process is similar to their previous work [8] and it classified ad non blind technique.

Anthony T.S. Ho et al from their work it is clear that that the simplicity of the fast Hadamard transformation not only offers a significant advantage in shorter processing time and ease of hardware implementation but also has more useful middle and high frequency bands available, for hiding the watermark [10]. This technique is robust to $60 \%$ of stir mark attacks. They 
described again how to embed a watermark in an image using fast Hadamard transformation [11]. To increase the invisibility of the watermark, a visual model based on original image characteristics, such as edges and textures are incorporated to determine the watermarking strength factor.

Roumen Kountchev et al discussed about how to embed a watermark based on decomposition with inverse difference pyramid and complex Hadamard transformation [12]. The features of this process are, it has no quantization values of transform coefficient, low computational complexity and different watermarking in every consecutive pyramid level. It deals about two types of watermarking. The technique proposed is robust against tampering, compression, affine transforms, filtration, dithering. In Tang Xianghong et al work watermark is embedded using singular value decomposition [13]. Cryptographic method can also be used to strengthen the secrecy of watermarking.

Emad E. Abdallah et al describes how to make a transparent and high rate embedding of watermarks into digital images using fast Hadamard transform and singular value decomposition [14]. The three main attractive features of their work are: high rate of watermark embedding into the cover image, robustness to the most common attacks, and possible implementation in real time. Elijah Mwangi describes a technique that embeds a grey level binary image as a watermark at selected DWT coefficients by the use of a private key [15]. This is achieved by spreading the binary image bits into the elements of a Hadamard matrix row by using CDMA techniques. The depth of watermarking is adjusted to give an image of acceptable quality. A correlation process is used to detect and recover the watermark. Their algorithm is effective against common signal processing attacks such as additive Gaussian noise, cropping, low pass filtering, and JPEG compression.

Bogdan J. Falkowski reveals about how to embed a watermark on the grey scale image using multi-resolution modified multipolarity Walsh-Hadamard transform and complex Hadamard transform [16]. The process is, the raw pixels are extracted from the bit map image and it is stored in two dimensional arrays. Then multi polarity Walsh-Hadamard transformation is applied to decompose the image into pyramid structure with various sub bands. The lowest frequency sub band is selected and segmented into $8 * 8$ blocks and then apply one dimensional complex Hadamard transformation on the rows followed by columns. Then complex Hadamard transform coefficients are altered and watermark is embedded. Then inverse complex Hadamard transformation and modified multi resolution Walsh-Hadamard transformation is applied and low frequency sub band is kept in the original position to get the watermarked image. This technique is robust to jpeg encoding, image resizing, dithering noise distortions, sharpening, cropping and successive watermarking.

Gaurav Bhatnagarl and Balasubramanian Raman describes about how to decompose an image using MR-WHT (Multiresolution Walsh-Hadamard Transform) and then middle singular values of High frequency sub-band at the finest and the coarsest level are modified with singular values of watermark [17]. The process of watermark embedding involves, Performing L-level MR-WHT on the host image and Selecting HH sub-band from the coarsest and the finest level and then Apply SVD on both HH sub-band and watermark image. Modify the middle singular values of the HH sub-band Perform inverse SVD to construct the watermarked Map modified sub-band to its original position and L-level inverse MR-WHT is performed to get the watermarked image.

Yasunori Ishikawa, Kazutake Uehira and Kazuhisa Yanaka tells about how to embed a illumination (invisibly contains watermark) using discrete cosine transformation and fast Hadamard transformation [18]. This technique is robust to illegal use of images of objects which has not watermarked. The data is embedded by two methods. First is, block method in which 1 bit data is embedded on one block and next is, majority method where same 1 bit is embedded in first three blocks sufficiently form one another. Thus improves the accuracy of reading the data.

Aris Marjuni, Rajasvaran Logeswaran, and M. F. Ahmad Fauzi proposed a watermarking scheme in which fast Walsh Hadamard transformation (FWHT) is applied on the original watermark before it is embedded on the DC coefficients of the host image [19]. The digital cosine transformation is applied to each $8 * 8$ block of original image to get the DC coefficient then embed the PN sequence and then apply the inverse discrete cosine transformation on DC component to reconstruct the watermarked image. It provides good visual perception and robust against common attacks. Yasunori Ishikawa, Kazutake Uehira, and Kazuhisa Yanaka proposed an "Illumination Watermarking" technology with which the images of objects without copyright protection can contain invisible digital watermarking [20]. The main attribute of this technology is watermarking can be added by light. The watermark is embedded by using fast Hadamard transformation and discrete cosine transformation. It has two methods to get a watermarked image. First is, by using two dimensional discrete cosine transformation and the next is, using two dimensional Hadamard transformation. It provides $100 \%$ accuracy and it is robust to jpeg compression.

From the survey of Hadamard transformation based watermarking techniques it is observed that the complexity of the transformation based watermarking work is greatly reduced as the transformation matrix have only +1 and -1 . In addition to that watermark embedded in Hadamard transform domain is more robust to many attacks. In this literature survey Hadamard transformation technique is combined with singular value decomposition (SVD) for embedding watermark. Multiresolution concept is used based on out HVS model. As a result in the proposed algorithm Hadamard transformation technique is combined with entropy model. This entropy model measure the information content of each block which is used as criterion for selection of blocks.

\section{OVERVIEW OF HADAMARD TRANSFORMATION}

The Hadamard transform is a non-sinusoidal, orthogonal transformation that decomposes a signal into a set of orthogonal, rectangular waveforms called Walsh functions. The transformation has no multipliers and is real because the amplitude of Walsh (or Hadamard) functions has only two values +1 or -1 
The Hadamard matrix is a square array of plus and minus ones whose rows (and columns) are orthogonal to one another. If $H$ is an $N \times N$ Hadamard matrix then the product of $H$ and its transpose is the identity matrix

Let $[U]$ represents the original image and $[V]$ the transformed image, the 2D-Hadamard transform is given by

$$
[V]=\left(H_{n}[U] H_{n}\right) / N
$$

Where $H$, represents an $N X N$ Hadamard matrix, $N=2^{n}, \mathrm{n}=1,2,3 \ldots$ with element values are either +1 or -1 . The advantage of Hadamard transform is that the elements of the transform matrix $H_{n}$, are binary real numbers. The inverse 2D-Hadamard transform (IHT) is given as

$$
[U]=H_{n}^{-1}[V] H_{n}{ }^{*}=\left(H_{n}[V] H_{n}\right) / N
$$

In the proposed algorithm, the transformation process is carried out on $8 \times 8$ blocks using the third order Hadamard transform matrix $\mathrm{H}_{3}$ which is given below.

$$
\mathrm{H}_{3}=\left[\begin{array}{rrrrrrrr}
1 & 1 & 1 & 1 & 1 & 1 & 1 & 1 \\
1 & -1 & 1 & -1 & 1 & -1 & 1 & -1 \\
1 & 1 & -1 & -1 & 1 & 1 & -1 & -1 \\
1 & -1 & -1 & 1 & 1 & -1 & -1 & 1 \\
1 & 1 & 1 & 1 & -1 & -1 & -1 & -1 \\
1 & -1 & 1 & -1 & -1 & 1 & -1 & 1 \\
1 & 1 & -1 & -1 & -1 & -1 & 1 & 1 \\
1 & -1 & -1 & 1 & -1 & 1 & 1 & -1
\end{array}\right]
$$

\section{PRELIMINARIES OF ENTROPY}

Entropy is a statistical measure of randomness that can be used to characterize the texture of the input image.

Let $\mathrm{P}$ contains the histogram counts. The entropy is represented as

$$
E=-\sum P \log 2(P)
$$

\section{PROPOSED ALGORITHM}

In the proposed algorithm, the Hadamard transformation technique and entropy concepts are used. The input image is divided into many blocks and entropy is calculated for each block. If the information content is very high then those blocks are marked as primer blocks for embedding watermark. The embedding and extraction process of watermark is given in Fig.1 and Fig.2. The Lena image of size $512 \times 512$ and watermark of size $64 \times 64$ is used to test the proposed system. The embedding and extraction algorithm is given below in detail.

\subsection{Watermark Embedding Algorithm}

1. Convert the cover image A into gray scale image if it is a color image.

2. Divide the cover image into blocks $B_{i j}$ of size $8 \times 8$

3. Find entropy $\mathrm{E}_{\mathrm{ij}}$ for each $8 \times 8$ blocks and find the threshold value $\mathrm{T}_{\mathrm{ij}}$.
4. Select the $8 \times 8$ blocks $\mathrm{B}_{\mathrm{ij}}{ }^{*}$ whose entropy value is greater than threshold value

5. Apply Hadamard Transformation to the selected $8 \times 8$ blocks $\mathrm{B}_{\mathrm{ij}}{ }^{*}=\left(\mathrm{H}_{\mathrm{n}}\left[\mathrm{B}_{\mathrm{ij}}{ }^{*}\right] \mathrm{H}_{\mathrm{n}}\right) / \mathrm{N}$..

6. Divide the watermark $\mathrm{W}$ into blocks $\mathrm{W}_{\mathrm{ij}}$ of size $8 \times 8$.Embed the watermarked block $\mathrm{W}_{\mathrm{ij}}$ with the selected block $\mathrm{B}_{\mathrm{ij}}{ }^{*}$ using constant factor.

8. Apply IHT to the embedded $8 \times 8$ blocks

$\mathrm{B}_{\mathrm{ij}}{ }^{*}=\mathrm{H}_{\mathrm{n}}^{-1}\left[\mathrm{~B}_{\mathrm{ij}}{ }^{*}\right] \mathrm{H}_{\mathrm{n}}{ }^{*}=\mathrm{B}_{\mathrm{ij}}{ }^{*}=\left(\mathrm{H}_{\mathrm{n}}\left[\mathrm{B}_{\mathrm{ij}}{ }^{*}\right] \mathrm{H}_{\mathrm{n}}\right) / \mathrm{N}$.

9. Rearrange the modified $8 \times 8$ blocks $\mathrm{B}_{\mathrm{ij}}{ }^{*}$ with the unmodified 8 $\mathrm{x} 8$ blocks $\mathrm{B}_{\mathrm{ij}}$.

10. Integrate the $8 \times 8$ blocks $\mathrm{B}_{\mathrm{ij}}$ to display the watermark image $\mathrm{A}^{*}$.

\subsection{Watermark Extraction Algorithm}

1. Convert the watermark image $A^{*}$ into gray scale image if it is a color image.

2. Divide the watermark image into blocks of size $8 \times 8 \mathrm{C}_{\mathrm{ij}}$.

3. Find entropy for each $8 \times 8$ blocks $\mathrm{E}_{\mathrm{ij}}{ }^{*}$ and find the threshold value $\mathrm{T}_{\mathrm{ij}}{ }^{*}$.

4. Select the $8 \times 8$ blocks $C_{i j}{ }^{*}$ whose entropy value is greater than threshold value.

5. Apply HT to the selected $8 \times 8$ blocks $\mathrm{C}_{\mathrm{ij}}{ }^{*}={ }^{*}=\left(\mathrm{H}_{\mathrm{n}}\left[\mathrm{C}_{\mathrm{ij}}{ }^{*}\right] \mathrm{H}_{\mathrm{n}}\right) / \mathrm{N}$.

6. Extract the watermark blocks $\mathrm{W}_{\mathrm{ij}}$ from the respective blocks $\mathrm{C}_{\mathrm{ij}}{ }^{*}$ using constant factor.

7. Apply IHT to the respective $8 \times 8$ blocks

$\mathrm{C}_{\mathrm{ij}}{ }^{*}=\mathrm{H}_{\mathrm{n}}{ }^{-1}\left[\mathrm{C}_{\mathrm{ij}}{ }^{*}\right] \mathrm{H}_{\mathrm{n}}{ }^{*}=\mathrm{C}_{\mathrm{ij}}{ }^{*}=\left(\mathrm{H}_{\mathrm{n}}\left[\mathrm{C}_{\mathrm{ij}}{ }^{*}\right] \mathrm{H}_{\mathrm{n}}\right) / \mathrm{N}$.

8. Rearrange the watermark blocks $\mathrm{W}_{\mathrm{ij}}$ and original $8 \times 8$ blocks $\mathrm{C}_{\mathrm{ij}}$.

9. Integrate the $8 \times 8$ blocks to display the original image $\mathrm{A}$ and watermark blocks to display the watermark image $\mathrm{W}$.

10. Convert to color image if needed and display the cover image.

\section{PERFORMANCE EVALUATION}

The performance of the algorithm is tested by simulating it using matlab software. Lena image of size $256 \times 256$ and watermark of size $64 \times 64$ is used to test its performance. The quality of the watermarked image is objectively measured using PSNR and it is observed that quality of the watermarked image is good. The measured psnr value is $42.7454 \mathrm{db}$. The original image, watermark and the watermarked image is shown in Fig.3. Similarly extracted watermark from undistorted watermarked image is shown in Fig.4. The similarity measure or the normalized correlation coefficient of original and extracted watermark from undistorted watermarked image is 1 . The psnr value is calculated using the eq. 4 . 


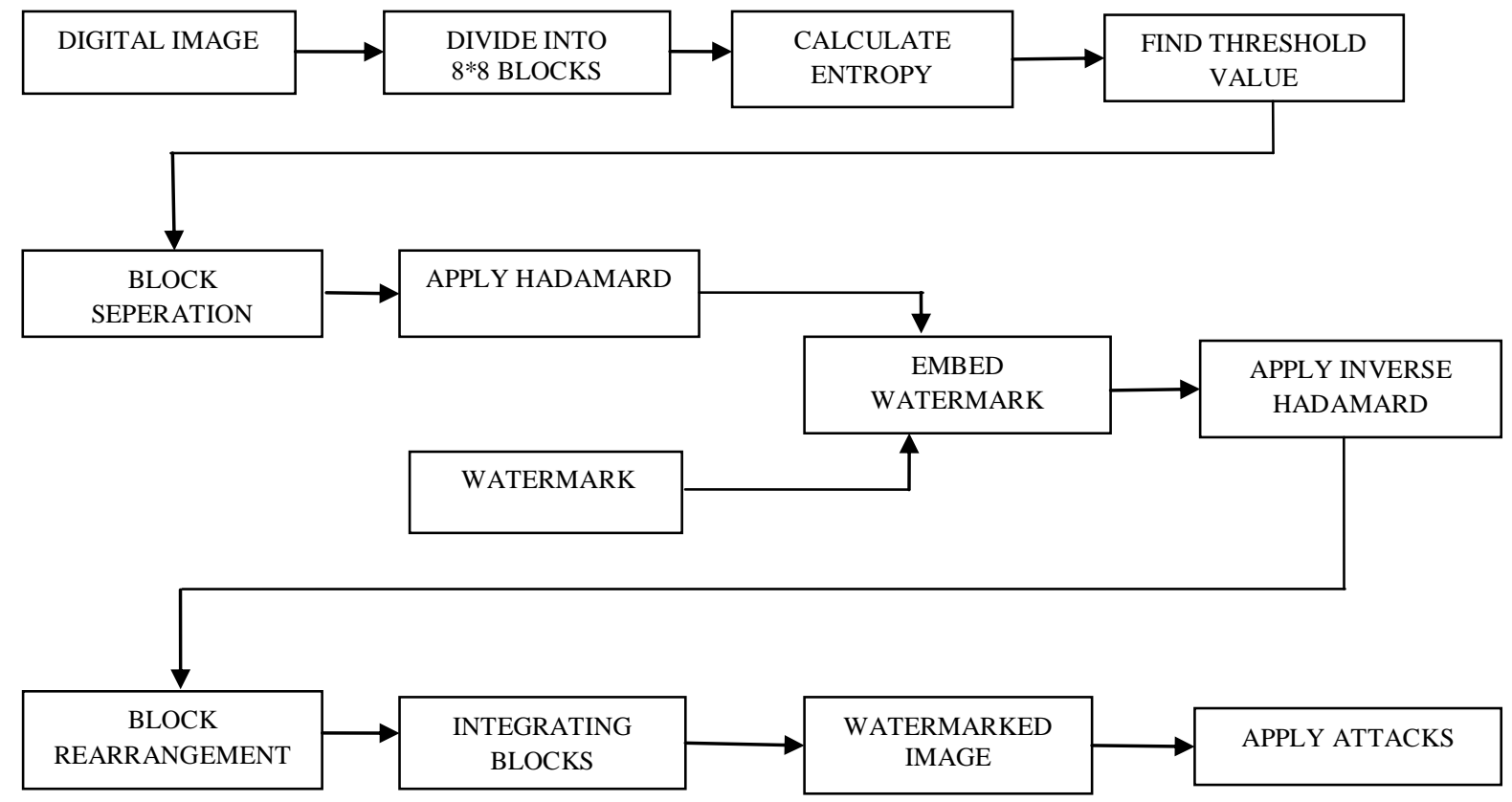

Figure.1 Embedding Process

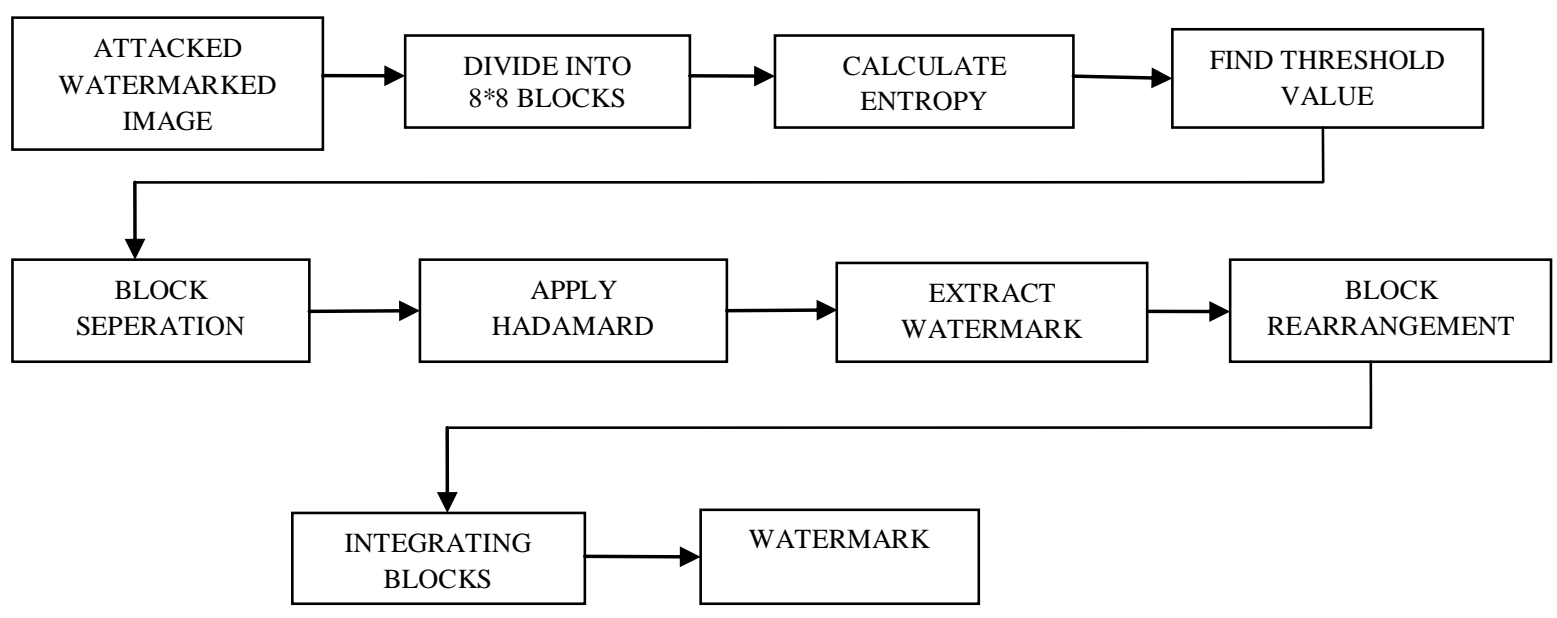

Figure.2 Extraction Process 
TABLE. 1 EXTRACTED WATERMARK FROM WATERMARKED IMAGE

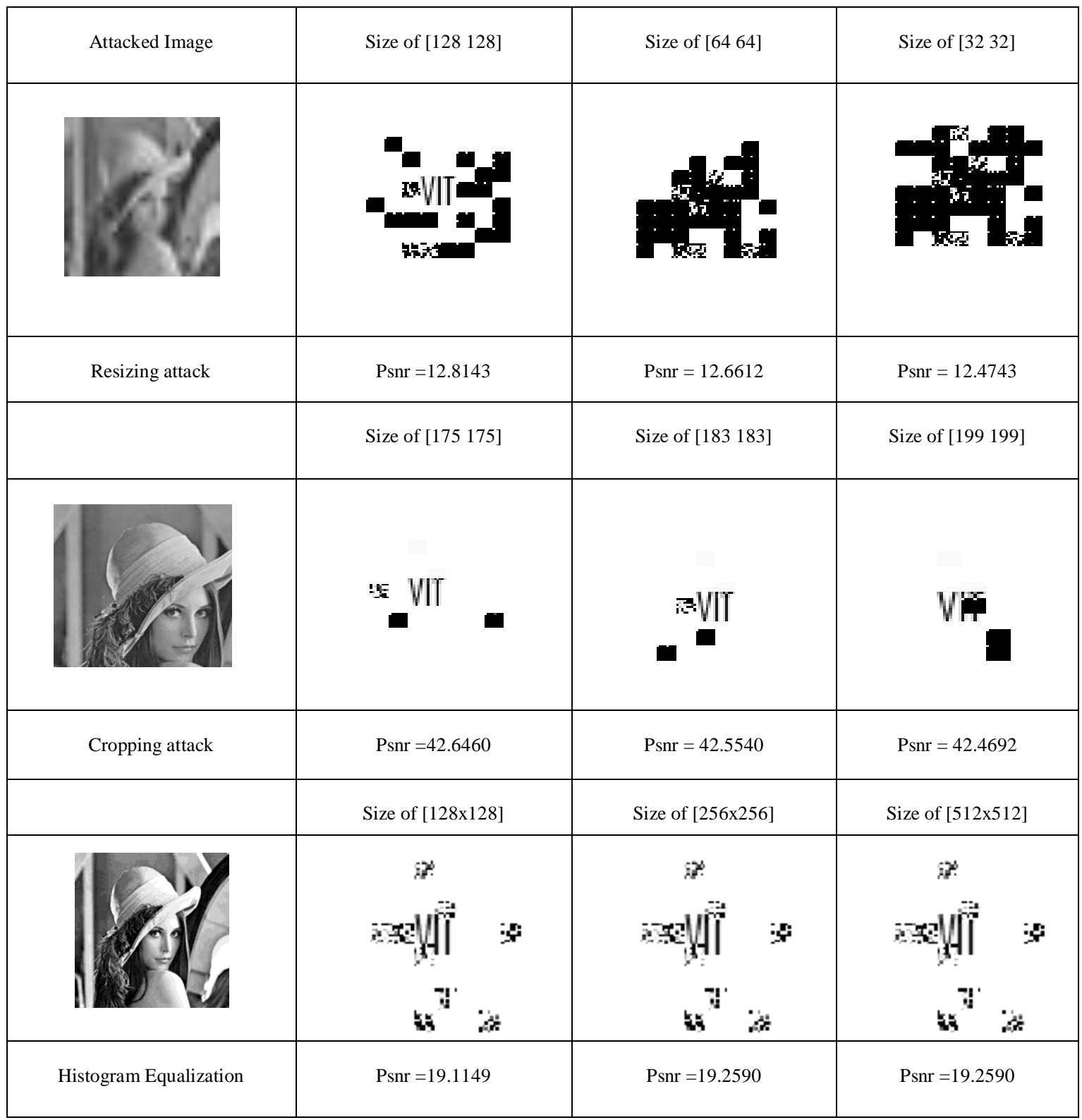


TABLE.2. EXTRACTED WATERMARK FROM WATERMARKED IMAGE

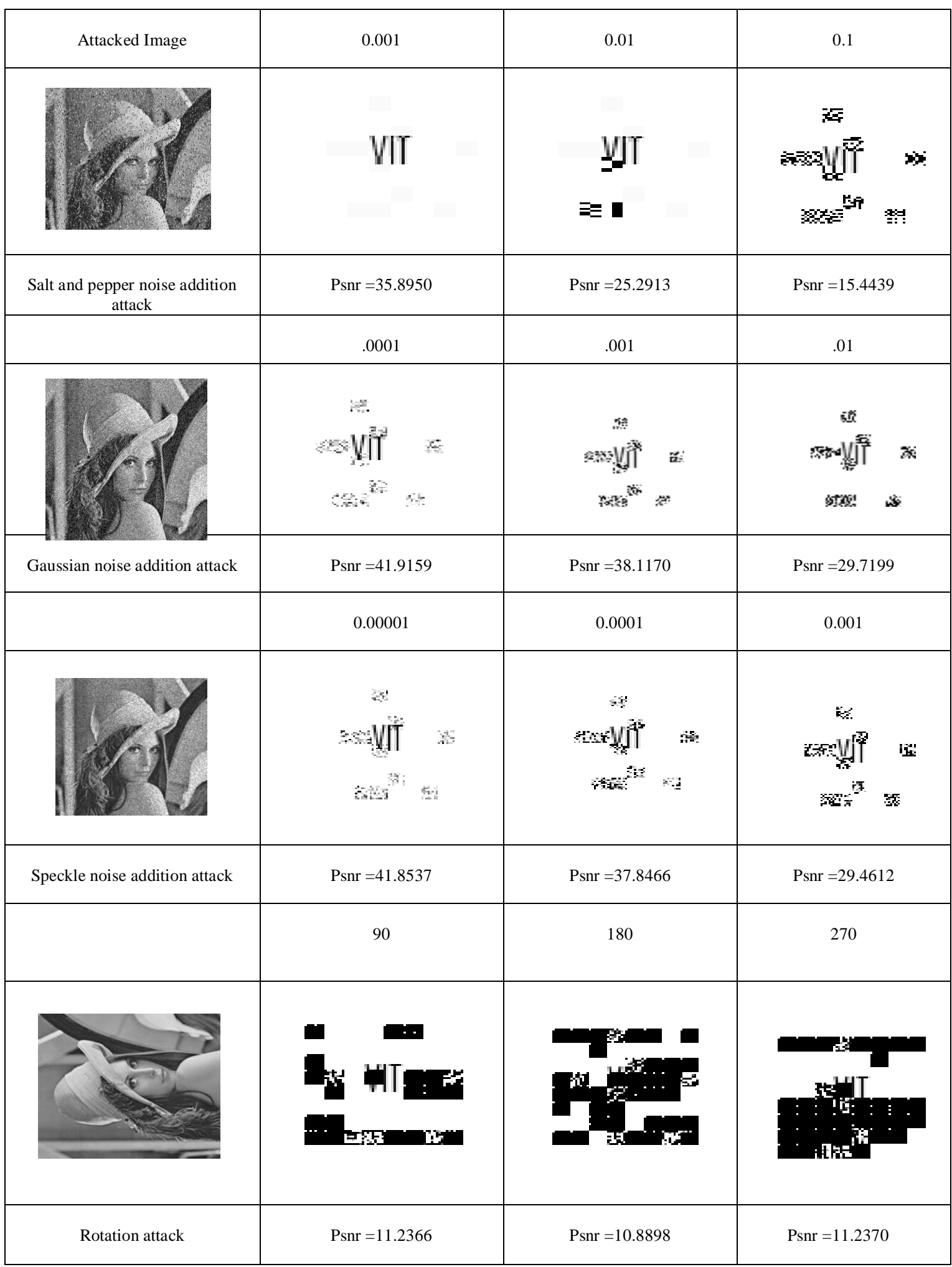




$$
\begin{aligned}
& M S E=\frac{1}{M N} \sum_{i=1}^{m} \sum_{j=1}^{n}(f(i, j)-f(i, j)) 2 \\
& P S N R=10 \log _{10}\left(\frac{255^{2}}{M S E}\right)
\end{aligned}
$$

$f(i, j)$ and $f^{\prime}(i, j)$ represent the pixel values of original host image and the watermarked image respectively and parameters $m, n$ specify row and column size of images respectively.

To test the robustness of the algorithm various attacks are introduced to the watermarked image. Initially the noise addition attack is introduced and it is observed that algorithm is robust if the variance is 0.001 and 0.01 . Various noises such as salt and pepper, Gaussian noise and speckle noise are added and robustness is tested. It is found that the algorithm is withstanding noise addition attacks. To check the quality of the extracted watermark from the distorted image the similarity measure is used. To check the similarity normalized correlation (NC) between original and extracted watermark is calculated using Eq. 7. If normalized correlation value is high then the quality of the extracted watermark is considered as good. If the vale of $\mathrm{NC}$ goes below $50 \%$, it is assumed that the algorithm is not robust to that particular attack.

$N C=\frac{\sum_{I=1}^{N} \sum_{J=1}^{M} W(I, J) * W_{Z}(I, J)}{\sqrt{\sum_{I=1}^{N} \sum_{J=1}^{M} W^{2}(I, J)} \sqrt{\sum_{I=1}^{N} \sum_{J=1}^{M} W^{2} Z(I, J)}}$

Algorithm is also tested with attacks like image resizing, cropping, rotation, image sharpening and histogram equalization. The tampered image and extracted watermark is shown in table 1 and table 2 .

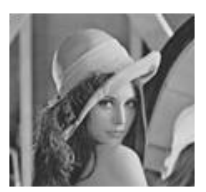

Fig.3 (a) CoverImage

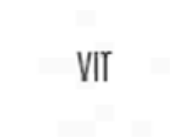

(b)Watermark

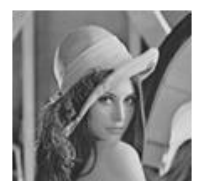

(c)Watermarkedimage

\section{CONCLUSION}

In this proposed algorithm Hadamard Transformation and entropy concept is used. As the Hadamard transformation matrix consists of +1 and -1 only the computational complexity is reduced. Embedding is carried out in most of the blocks which have more information content which is measured through entropy equation. The proposed algorithm is found to be robust to most of the image related attacks as per the results. So it is classified as non-blind watermarking algorithm which can be used for copyright protection where robustness is very important. Finally, we note that our experimental results suggest that our proposed algorithm is not robust to rotation attack. Thus our future work is to make algorithm robust to rotation attack.

\section{REFERENCES}

[1] F.Hartung and M. Kutter, "Multimedia Watermarking Techniques," in proc. of the IEEE, vol. 87, no. 7, pp. 10791107, July 1999.

[2] Ali Al-Haj "Combined DWT-DCT Digital Image Watermarking," Journal of computer science 3 (9),740-746, ISSN, 2007.

[3] W. Bender, D. Gruhl, N. Morimoto and A. Lu, "Techniques for data hiding," IBM Systems Journal, vol.35, no. 3\&4, pp. 313-336, 1996.

[4] I. J. Cox, J. Killian, F. T. Leighton and T. Shamoon,"Secure spread spectrum watermarking for multimedia,"IEEE Transactions on Image Processing, vol. 6, no. 12,pp. 16731687, December 1997.

[5] J. J. K. 0 Ruanaidh, W. J. Dowling and F. M. Boland,"Watermarking digital images for copyright protection,'IEE Proceedings - Vision, Image and Signal Processing, vol. 143, no. 4, pp. 250-256, August 1996.

[6] M. D. Swanson, M. Kobayashi and A. H. Tewfik, "Multimedia data-embedding and watermarking techniques," Proceedings of the IEEE, vol. 86, no. 6, pp.1064-1087, June 1998.

[7] Navas K A,Ajay Mathews Cheriyan, Lekshmi. M, Archana Tampy.S, Sasikumar M, "DWT-DCT-SVD Based Watermarking," 3rd International Conference on Communication Systems Software and Middleware and Workshops, 2008.

[8] B. J. Fnlkowski and Lip-San Lim,'Image watermarking using Hadamard Transforms", Electronics Letters, vol. 36, no. 3, pp. 211-213, February 2000.

[9] Bogdan J. Falkowski and Lip-San Lim,'Image Watermarking Using the Complex Hadamard Transform", IEEE International Symposium on Circuits and Systems, vol. 4, pp. 573-576, Geneva, Switzerland, May 2000.

[10] Anthony T.S. Ho, Jun Shen, Soon Hie Tan, Alex C. Kot,'Digital Image-in-Image Watermarking For Copyright Protection Of Satellite Images Using the Fast Hadamard Transform", IEEE International Geosciences and Remote Sensing Symposium, vol. 6, pp. 3311-3313, 2002.

[11] Anthony T.S. Ho, Jun Shen, Andrew K.K. Chow, Jerry Woon," Robust digital image-image watermarking algorithm using fast Hadamard transform", International Symposium on circuits and systems, vol. 3, pp. III-826-III829, May 2003.

[12] Roumen Kountchev Mariofanna Milanova Charles Ford Stuart Rubin,"Multimedia Watermarking with complex Hadamard transform in the inverse pyramidal 
decomposition", International conference on Information Reuse and Integration, pp. 305-310, October 2003.

[13] Tang Xianghong, Yang Lianjie, YueHengli, Yin Zhongke,"A Watermarking Algorithm Based on the SVD and Hadamard Transform", International Conference on Communications, circuits and system, vol. 2-877, May 2005.

[14] Emad E. Abdallah, A. Ben Hamza, and Prabir Bhattacharya,"Robust block based image watermarking scheme using fast Hadamard transformation and single value decomposition", The 18th International Conference on Pattern Recognition, vol. 3, pp. 673-676, 2006.

[15] Elijah Mwangi,"A wavelet based image watermarking scheme with a cdma/Hadamard embedding technique", $9^{\text {th }}$ International Symposium on Signal Processing and its Applications, pp. 1-4, February 2007.

[16] Bogdan J. Falkowski,"Multi-Polarity Complex Hadamard Transforms for Phase Watermarking Algorithm", $6^{\text {th }}$ International Conference on Information Communication and Signal Processing, pp. 1-5, December 2007.
[17] Gaurav Bhatnagarl and Balasubramanian Raman2,'RobustWatermarking in Multiresolution WalshHadamard Transform", International Advance Computing Conference (IACC 2009), Patiala, India, pp. 894-899, March 2009.

[18] Yasunoriishikawa, Kazutakeuehiraand Kazuhisa yanaka,'Illumination Watermarking Technique Using Orthogonal Transforms", Industry Applications society Annual Meeting, pp. 1-4, October 2009.

[19] Aris Marjuni, Rajasvaran Logeswaran, M. F.Ahmad Fauzi,"An image watermarking scheme based on fast Walsh Hadamard transformation and discrete Cosine transformation", International Conference on Networking and Information Technology, pp. 289-293, June 2010.

[20] Yasunori Ishikawa, Kazutake Uehira, and Kazuhisa Yanaka,"Practical evaluation of illumination watermarking technique using orthogonal transforms", Journal of Display Technology, vol. 6, no. 9, pp. 351-358, September 2010. 